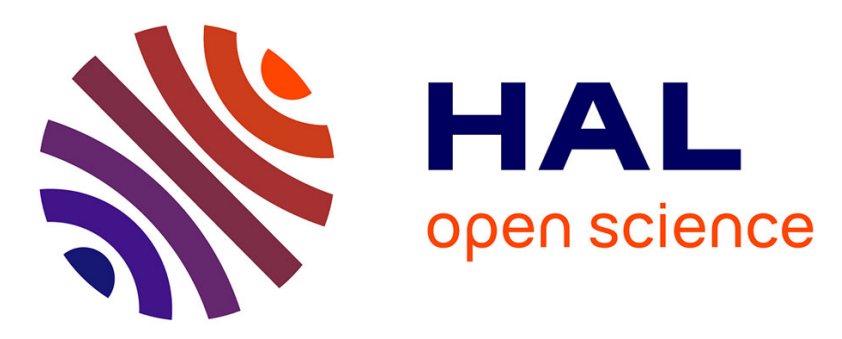

\title{
Ultrafast barrierless photoisomerization and strong ultraviolet absorption of photoproducts in plant sunscreens
}

Jian Luo, Yan Liu, Songqiu Yang, Amandine Flourat, Florent Allais, Keli Han

\section{- To cite this version:}

Jian Luo, Yan Liu, Songqiu Yang, Amandine Flourat, Florent Allais, et al.. Ultrafast barrierless photoisomerization and strong ultraviolet absorption of photoproducts in plant sunscreens. Journal of Physical Chemistry Letters, 2017, 8 (5), pp.1025-1030. 10.1021/acs.jpclett.7b00083 . hal-01604320

\author{
HAL Id: hal-01604320 \\ https://hal.science/hal-01604320
}

Submitted on 26 May 2020

HAL is a multi-disciplinary open access archive for the deposit and dissemination of scientific research documents, whether they are published or not. The documents may come from teaching and research institutions in France or abroad, or from public or private research centers.
L'archive ouverte pluridisciplinaire HAL, est destinée au dépôt et à la diffusion de documents scientifiques de niveau recherche, publiés ou non, émanant des établissements d'enseignement et de recherche français ou étrangers, des laboratoires publics ou privés.

$$
\text { Copyright }
$$




\section{Letter}

\section{Ultrafast Barrierless Photoisomerization and Strong UV Absorption of Photoproducts in Plant Sunscreens} Jian Luo, Yan Liu, Songqiu Yang, Amandine F. Flourat, Florent Allais, and Keli Han

J. Phys. Chem. Lett., Just Accepted Manuscript • DOI: 10.1021/acs.jpclett.7b00083 • Publication Date (Web): 08 Feb 2017

Downloaded from http://pubs.acs.org on February 10, 2017

\section{Just Accepted}

"Just Accepted" manuscripts have been peer-reviewed and accepted for publication. They are posted online prior to technical editing, formatting for publication and author proofing. The American Chemical Society provides "Just Accepted" as a free service to the research community to expedite the dissemination of scientific material as soon as possible after acceptance. "Just Accepted" manuscripts appear in full in PDF format accompanied by an HTML abstract. "Just Accepted" manuscripts have been fully peer reviewed, but should not be considered the official version of record. They are accessible to all readers and citable by the Digital Object Identifier (DOI®). "Just Accepted" is an optional service offered to authors. Therefore, the "Just Accepted" Web site may not include all articles that will be published in the journal. After a manuscript is technically edited and formatted, it will be removed from the "Just Accepted" Web site and published as an ASAP article. Note that technical editing may introduce minor changes to the manuscript text and/or graphics which could affect content, and all legal disclaimers and ethical guidelines that apply to the journal pertain. ACS cannot be held responsible for errors or consequences arising from the use of information contained in these "Just Accepted" manuscripts.

\section{ACS Publications}




\section{Ultrafast Barrierless Photoisomerization and Strong}

\section{UV Absorption of Photoproducts in Plant}

\section{Sunscreens}

JianLuo, ${ }^{\dagger \ddagger \#}$ Yan Liu, ${ }^{\dagger \#}$ Songqiu Yang, ${ }^{\dagger}$ Amandine L. Flourat,,${ }^{\| " \pi}$ Florent Allais,

${ }^{\dagger}$ State Key Laboratory of Molecular Reaction Dynamics, Dalian Institute of Chemical Physics

(DICP), Chinese Academy of Sciences, 457 Zhongshan Road, Dalian, Liaoning 116023, China

${ }^{\ddagger}$ University of the Chinese Academy of Sciences, Beijing 10049, China

"Chaire Agro-Biotechnologies Industrielles (ABI), AgroParisTech, CEBB 3 rue des Rouges Terres 51110 Pomacle, France

§UMR GMPA, INRA/AgroParisTech/CNRS/Université Paris-Saclay, Avenue Lucien Brétignières 78850 Thiverval-Grignon, France

"Institut Jean-Pierre Bourgin, INRA/AgroParisTech/CNRS/Université Paris-Saclay, Route de Saint-Cyr 78026 Versailles, France

\section{AUTHOR INFORMATION}

Corresponding Author

*Keli Han: klhan@dicp.ac.cn 


\begin{abstract}
Sunscreens are aimed at protecting skins from solar UV irradiation. By utilizing femtosecond transient absorption spectroscopy and time-dependent density functional theory, we explain nature's selection of sinapoyl malate rather than sinapic acid as the plant sunscreen molecule. In physiological $\mathrm{pH}$ condition, the two molecules are deprotonated and their excited $\pi \pi^{*}$ states are found to relax to the ground states in a few tens of picoseconds via a barrierless trans-cis photoisomerization. After forming the cis-photoproduct, the efficacy of sinapic acid is reduced much. In contrast, the efficacy of sinapoyl malate is affected slightly because the cisproduct still absorbs UV light strongly. In addition, protonated sinapic acid is found to be a good potential sunscreen molecule.
\end{abstract}

\title{
TOC GRAPHICS
}

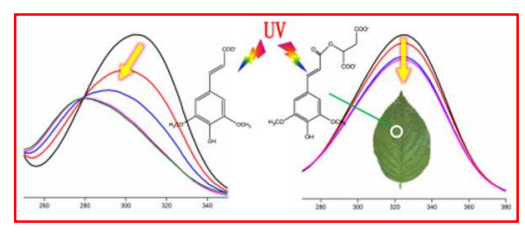

KEYWORDS Femtosecond, TDDFT, Sinapic Acid, Photochemistry, Excited State. 
An over exposure to ultraviolet (UV) radiation has deleterious effects on animals and higher plants, and with the depletion of the ozone layer due to the increasing pollution, this exposure of UV is increasing day by day. ${ }^{1-3}$ Various sunscreens are designed to protect skins from damages by UV light, such as oxybenzone ${ }^{4}$ and cinnamate-based molecules. ${ }^{5-7}$ A good sunscreen agent should have a large absorption section on UVB (285-320 nm) and UVA (320-400 nm) light and dissipate the energy into surroundings efficiently. ${ }^{8}$ Plants sustain much longer exposure to UV radiation than animals. As a result, nature has selected efficient screening agents to protect plants. In adult Arabidopsis plants, sinapoyl malate is deposited in the leaf epidermis to prevent penetration of UV radiation. ${ }^{9-11}$ Sinapoyl malate is an ester derivative of sinapic acid. Both of them can efficiently dissipate the energy into their surroundings. ${ }^{12}$ As a result, an interesting question is why nature selects sinapoyl malate rather than sinapic acid as the plant sunscreen?

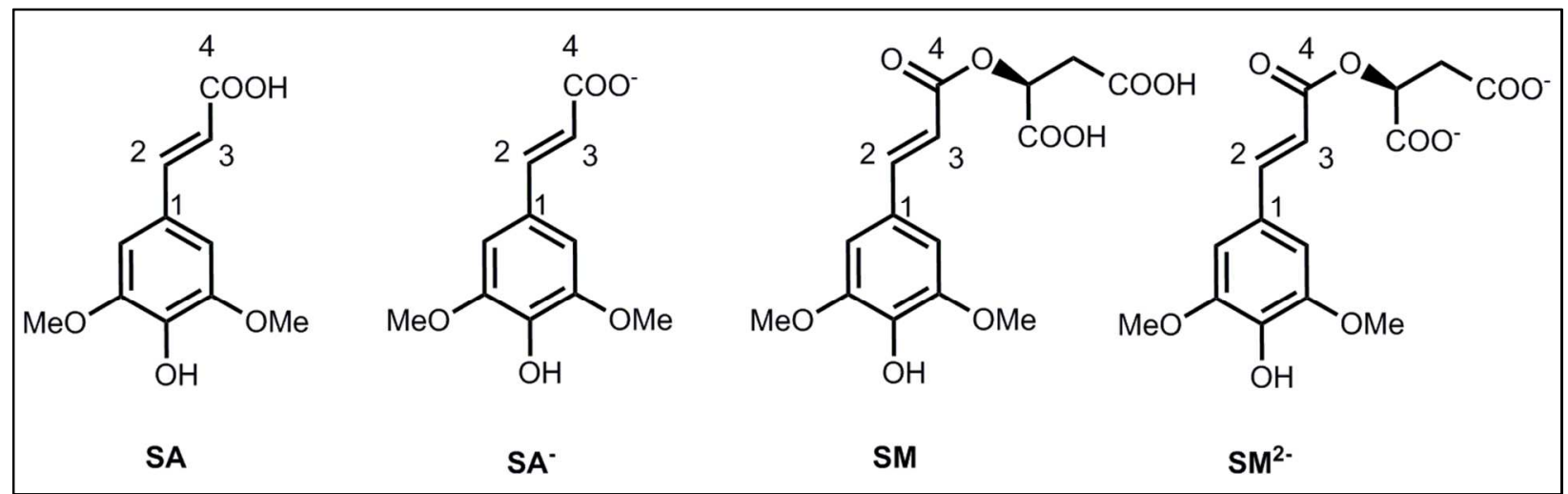

Figure 1. Chemical structures of sinapic acid in acidic environment (SA) and neutral environment ( $\left.\mathrm{SA}^{-}\right)$, as well as the chemical structures of sinapoyl malate in acidic environment (SM) and neutral environment $\left(\mathrm{SM}^{2-}\right)$.

Sinapoyl malate in the gas phase exhibits a more broadening absorption spectrum in the UVB region than sinapic acid, which is suggested to be the reason for nature's selection of sinapoyl 
malate as the sunscreen molecule. ${ }^{13}$ However, in dioxane, acetonitrile and methanol, the two molecules both exhibit high extinction coefficients in the UVB region and thus the reason for the selection is still under discussion. ${ }^{12}$ In addition, the excited-state relaxation mechanisms of sinapic acid and sinapoyl malate in the gas phase differ from that in organic solvents. In the gas phase, the initially populated $\pi \pi^{*}$ states are suggested to relax to long-lived $n \pi^{*}$ states through internal conversion. ${ }^{13}$ However, a femtosecond study suggests that in organic solvents the $\pi \pi^{*}$ states relax to the ground state $\left(\mathrm{S}_{0}\right)$ via an ultrafast trans-cis photoisomerization. ${ }^{12}$ The trans-cis photoisomerization is also found in the relaxations of the closely related para-methoxy methylcinnamate (p-MMC) in aqueous solution, ${ }^{5,6}$ as well as sinapic acid in aqueous solution. ${ }^{14}$ However, the relaxation mechanism of sinapoyl malate in aqueous solution is unknown.

Herein, we explore the excited-state dynamics of sinapic acid and sinapoyl malate in aqueous solution. In $\mathrm{pH}=6 \sim 8$ aqueous solution, the carboxyl group is deprotonated and the phenolic hydroxyl group is protonated. ${ }^{15}$ Thus, sinapic acid and sinapoyl malate exist as $\mathrm{SA}^{-}$and $\mathrm{SM}^{2-}$ species, respectively, as shown in Figure 1. The protonated SA species is also studied under acidic environment in order to provide a guideline for the chemical modification of sinapic acid, such as methyl sinapate. ${ }^{12,13}$ The potentials of $\mathrm{SA}, \mathrm{SA}^{-}$and $\mathrm{SM}^{2-}$ as sunscreen molecules are investigated using static absorption and femtosecond transient absorption (FTA) spectroscopy, as well as time-dependent density functional theory (TD-DFT). Note that the abbreviation SA only represents the protonated form of sinapic acid in acidic environment. The ultrafast barrierless photoisomerization of the three species are identified. The electronic absorption properties of their cis-photoproducts are also discussed.

Sinapic acid ( $\geq 98 \%$, Sigma-Aldrich) was dissolved in water and hydrochloric acid $(\sim 1 \mathrm{mM})$ to obtain the $\mathrm{SA}^{-}$and $\mathrm{SA}$ aqueous solution, respectively. Sinapoyl malate was synthesized as 
described in previous studies. ${ }^{16}$ The high-purity powder was dissolved in water and thus the $\mathrm{SM}^{2-}$ aqueous solution was obtained. The three species were confirmed by UV-Vis absorption spectroscopy measurements (Perkin-Elmer). The FTA setup was used as described in previous studies. ${ }^{17,18}$ Briefly, the pump pulse was $320 \mathrm{~nm}$ for SA, $305 \mathrm{~nm}$ for $\mathrm{SA}^{-}$, and $325 \mathrm{~nm}$ for $\mathrm{SM}^{2-}$. The powers of these excitation pulses were about $0.5 \mathrm{~mW}$. Probe pulse was drawn from a broadband white-light continuum (400-700 nm), generated by focusing a weak $800 \mathrm{~nm}$ pulse into a sapphire. The polarization of the pump pulse was set to the magic angle $\left(54.7^{\circ}\right)$ relative to the probe pulse. To avoid the concentration of cis-products induced by UV excitation, transient absorption spectra were taken using a flow cell. The UV-Visible spectra before and after FTA measurements were shown in Figure S1 (see the supporting information, SI). Continuous UV irradiation studies were performed on the three solutions using a nanosecond $355 \mathrm{~nm}$ pulse with the power of $3 \mathrm{~mW}$ in a $10 \mathrm{~mm} * 10 \mathrm{~mm}$ quartz cuvette. The $355 \mathrm{~nm}$ pulse was chosen because it was near the center of UVA light. The UV-Vis absorption spectra were taken to probe the changes upon photoexcitation.

All the ab initio calculations were performed using the Gaussian09 program. ${ }^{19}$ Default parameters were used unless otherwise indicated. TD-DFT with the M062X functional ${ }^{20}$ was employed, combined with the linear-response polarized continuum model (LR-PCM) ${ }^{21,22}$ and 6$31+\mathrm{g}(\mathrm{d}, \mathrm{p})$ basis set. All the optimized ground-state and excited-state geometries were confirmed by frequency calculations. Trans-cis photoisomerization paths were constructed by linearly interpolated internal coordinate (LIIC) method at the LR-PCM/TD-M062X/6-31+g(d, p) calculation level. Further details could be found in the SI. 


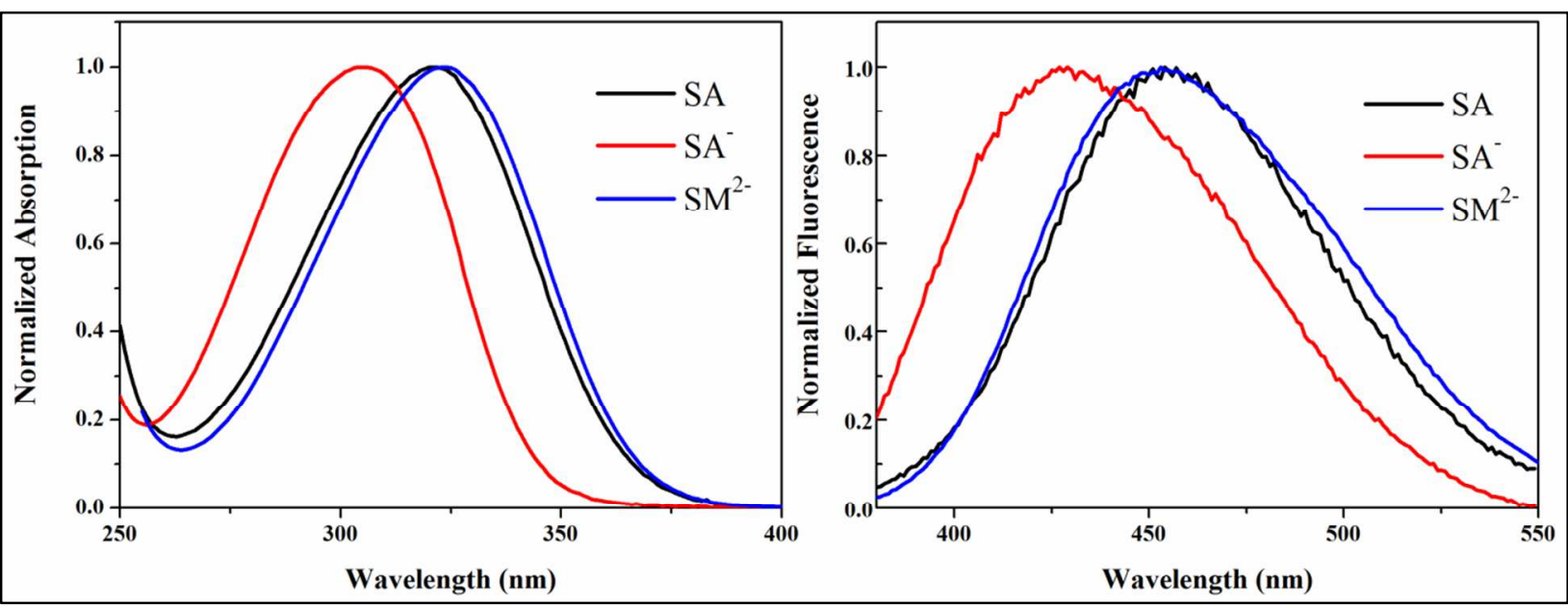

Figure 2.Normalized steady-state absorption (left) and fluorescence (right) spectra of SA, $\mathrm{SA}^{-}$ and $\mathrm{SM}^{2-}$ in aqueous solution.

The steady-state UV-Vis absorption and fluorescence spectra of SA, $\mathrm{SA}^{-}$and $\mathrm{SM}^{2-}$ are shown in Figure 2. All the three species have large absorption section at UVB and high-energy UVA regions. The absorption spectrum of $\mathrm{SA}^{-}$is peaked at $304 \mathrm{~nm}$ and its intensity decreases to zero at approximately $360 \mathrm{~nm}$. Comparatively, the absorption spectra of SA and $\mathrm{SM}^{2-}$ are slightly red shifted. Their absorption maxima are both at $320 \mathrm{~nm}$ and they absorb UVA light till approximately $380 \mathrm{~nm}$. The more broadening absorption of $\mathrm{SA}$ and $\mathrm{SM}^{2-}$ relative to $\mathrm{SA}^{-}$makes them behave as better sunscreen molecules. The fluorescence spectra of SA and $\mathrm{SM}^{2-}$ are both peaked at approximately $450 \mathrm{~nm}$, also slightly red shifted compared to the fluorescence spectrum of $\mathrm{SA}^{-}$, which is peaked at approximately $425 \mathrm{~nm}$. The deprotonation state of sinapic acid affects its electronic absorption characteristics. ${ }^{14}$ For example, deprotonating the carboxylic group of sinapic acid results in the absorption maximum blue shifted slightly from 320 to $304 \mathrm{~nm}$. In contrast, further deprotonating the phenolic hydroxyl group ( $\left.\mathrm{SA}^{2-}\right)$ makes the absorption maximum red shifted largely to $350 \mathrm{~nm}$, as shown in Figure S2. This implies that deprotonating the carboxylic group and phenolic hydroxyl group have exactly the opposite effects on the 
electronic excitation energy of sinapic acid. This phenomenon is also observed in sinapoyl malate. Deprotonating the carboxyl group of sinapoyl malate only leads to the absorption maximum blue shifted slightly from 328 to $320 \mathrm{~nm}$, while further deprotonating the phenolic hydroxyl group $\left(\mathrm{SM}^{3-}\right)$ leads to the absorption maximum largely red shifted to $380 \mathrm{~nm}$ (Figure $\mathrm{S} 2$ ). The small absorption cross section in UVB region makes $\mathrm{SA}^{2-}$ and $\mathrm{SM}^{3-}$ not suitable to serve as sunscreen molecules.

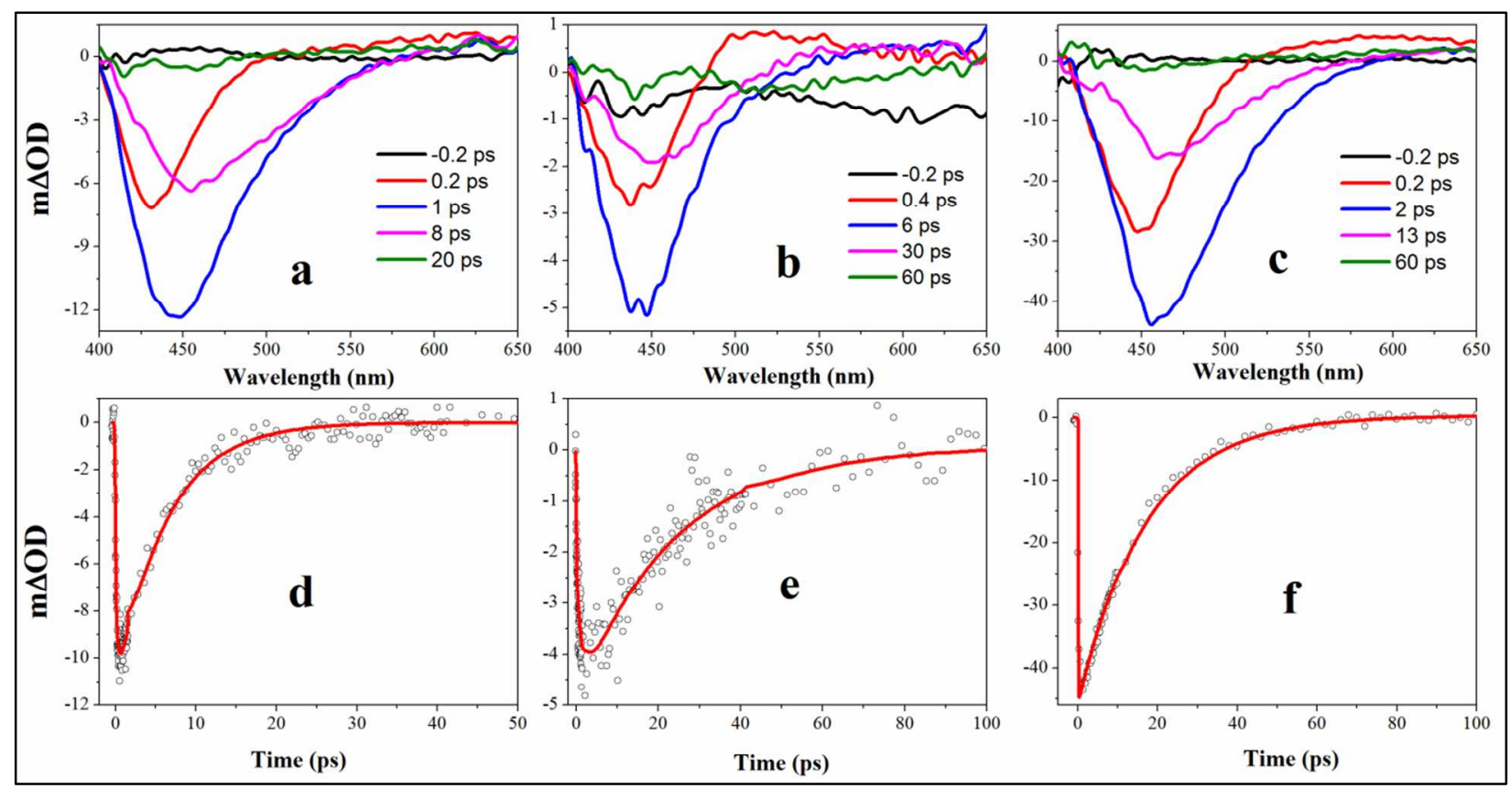

Figure 3. Femtosecond wavelength evolution curves of (a) $\mathrm{SA}$, (b) $\mathrm{SA}^{-}$, and (c) $\mathrm{SM}^{2-}$. Fitting results of the decay of (d) $\mathrm{SA}$ at $430 \mathrm{~nm}$, (e) $\mathrm{SA}^{-}$at $430 \mathrm{~nm}$, and (f) $\mathrm{SM}^{2-}$ at $453 \mathrm{~nm}$. Black circles represent the experimental data and red lines represent the fitting curves.

Figure 3 shows the FTA spectra of SA, $\mathrm{SA}^{-}$and $\mathrm{SM}^{2-}$ for photoexcitation at 320, 305 and 325 $\mathrm{nm}$, respectively. For SA, the FTA spectra are dominated by a broad feature spanning the spectral region of $\sim 400-500 \mathrm{~nm}$ centered at $450 \mathrm{~nm}$. This is attributed to the stimulated emission, which is in line with the steady-state fluorescence (Figure 2). ${ }^{12-14}$ In this region, the absorption of 
solvated electron and phenoxyl-type radical species can be ignored due to the strong absorption intensity of solutes (Figure S1) and much shorter lifetime relative to the radicals. ${ }^{14}$ At 0.2 ps, the stimulated emission is peaked at $\sim 430 \mathrm{~nm}$ and then it red shifts to $\sim 450 \mathrm{~nm}$ at $1 \mathrm{ps}$, induced by the intramolecular vibrational energy redistribution (IVR) and solvation relaxation. The negative absorption finally decays to the baseline by $\sim 20 \mathrm{ps}$. The FTA spectra for $\mathrm{SA}^{-}$and $\mathrm{SM}^{2-}$ are also dominated by their negative stimulated emissions. Obviously, $\mathrm{SA}^{-}$decays slower than the other two molecules. To give a quantitative insight into their dynamical relaxations, the decays of their stimulated emissions are fitted by mono- or bi-exponential functions. The lifetimes of the three species are summarized in Table 1. A bi-exponential function fits well the decays of SA and SA'. In SA, the $\tau_{1}(0.28 \mathrm{ps})$ with a positive pre-exponential factor is assigned to the coupled IVR and solvation relaxation processes, while the $\tau_{2}(6.1 \mathrm{ps})$ with a negative pre-exponential factor is assigned to the decay of $\pi \pi^{*}$ state. The $\pi \pi^{*}$ state might relax to a dark $n \pi^{*}$ state like in the gas phase and/or decay back to the $\mathrm{S}_{0}$ state via trans-cis photoisomerization like cinnamate derivatives, ${ }^{6,13}$ which will be discussed further by ab initio calculations. In addition, the assignment strategy is also reasonable for the fitted lifetimes of $\mathrm{SA}^{-}$. The $23.5 \mathrm{ps}$ lifetime of $\mathrm{SA}^{-}$ is in good agreement with the 26 ps lifetime measured by Vengris et al. ${ }^{14}$ The $\tau_{1}$ of $\mathrm{SA}^{-}(1.1 \mathrm{ps})$ is much longer than that of SA $(0.28 \mathrm{ps})$, indicating that the IVR and solvation relaxation processes are much slower for $\mathrm{SA}^{-}$. This can be also seen from their wavelength evolutions (Figures $3 \mathrm{a}, 3 \mathrm{~b}$ ). The decay of $\mathrm{SM}^{2-}$ is fitted by a mono-exponential function and $\tau_{1}=16.9 \mathrm{ps}$ is obtained, which corresponds to the decay of $\pi \pi^{*}$ state. The IVR and solvation relaxation processes are absent in the fitting results. Based on the similar wavelength evolutions of SA and $\mathrm{SM}^{2-}$ (Figures $3 \mathrm{a}, 3 \mathrm{c}$ ), it can be inferred that the lifetime of IVR and solvation relaxation process is at sub-ps timescale. Although the $\pi \pi^{*}$ state relaxation of $\mathrm{SA}^{-}$is the slowest, it cannot conclude 
that $\mathrm{SA}^{-}$is not a suitable sunscreen molecule, because the lifetimes of the other two molecules are also at the same timescale. There should be other reasons for nature's selection of $\mathrm{SM}^{2-}$ as the sunscreen molecule.

Table 1. Summary of the excited-state lifetimes of SA, $\mathrm{SA}^{-}$and $\mathrm{SM}^{2-}$ based on their decays of stimulated emissions

$\begin{array}{cccc} & \mathrm{SA} & \mathrm{SA}^{-} & \mathrm{SM}^{2-} \\ \mathrm{a}_{1} & 0.46 \pm 0.08 & 0.27 \pm 0.05 & -46.3 \pm 1.0 \\ \tau_{1} / \mathrm{ps} & 0.28 \pm 0.09 & 1.1 \pm 0.4 & 16.9 \pm 1.1 \\ \mathrm{a}_{2} & -1.14 \pm 0.03 & -0.49 \pm 0.04 & \\ \tau_{2} / \mathrm{ps} & 6.1 \pm 0.4 & 23.5 \pm 3.0 & \end{array}$

Table 2. Vertical excitation energies (in eV) including the first and second excited states $\left(\mathrm{S}_{1}, \mathrm{~S}_{2}\right)$ of trans- SA, $\mathrm{SA}^{-}$and $\mathrm{SM}^{2-}$ at the LR-PCM/TD-M062X/6-31+g(d, p) level, as well as the $\mathrm{S}_{1}$ state of their respective cis- forms. Oscillator strengths are shown in the parentheses.

\begin{tabular}{cccc} 
& $\mathrm{E}(\mathrm{SA}) / \mathrm{eV}$ & $\mathrm{E}\left(\mathrm{SA}^{-}\right) / \mathrm{eV}$ & $\mathrm{E}\left(\mathrm{SM}^{2-}\right) / \mathrm{eV}$ \\
\hline trans-S & $4.05(0.69)$ & $4.46(0.64)$ & $4.12(0.73)$ \\
trans-S & $4.51(0.03)$ & $4.73(0.00)$ & $4.62(0.02)$ \\
cis-S $_{1}$ & $4.03(0.57)$ & $4.78(0.46)$ & $4.25(0.39)$
\end{tabular}

In order to determine whether the initially populated $\pi \pi^{*}$ state decays to dark n $\pi^{*}$ state or the $\mathrm{S}_{0}$ state via trans-cis photoisomerization, TD-DFT calculations are carried out. The $\mathrm{S}_{0}$ geometries of trans-SA, $\mathrm{SA}^{-}$and $\mathrm{SM}^{2-}$ are optimized, as shown in Figure $\mathrm{S} 3$. The dihedral angle $\eta\left(\mathrm{C}_{1}-\mathrm{C}_{2^{-}}\right.$ $\mathrm{C}_{3}-\mathrm{C}_{4}$ ), defined to describe the double bond rotation, is $180^{\circ}$ for all the three species. Based on 
these equilibrium geometries, the vertical excitation energies and oscillator strengths of the $\mathrm{S}_{1}$ and $\mathrm{S}_{2}$ were calculated at the LR-PCM/TD-M062X/6-31+g(d, p) level, as collected in Table 2. For all the trans-species, the $\mathrm{S}_{1}$ state is bright $\pi \pi^{*}$ state and the $\mathrm{S}_{2}$ state is dark $n \pi^{*}$ state. After considering the intermolecular hydrogen bonding interactions with water, the results do not have significant changes, as shown in Figure S4 and Table S1. This energy level sequence is contrast with the case in the gas phase due to the strong polar aqueous environment stabilizing the $\pi \pi^{*}$ state and destabilizing the $n \pi^{*}$ state. ${ }^{5,13}$ The calculated excitation energies are overestimated compared to the experimental absorption maxima. However, the calculated results are in qualitative agreement with experiment. The electronic absorption energy of trans-SA ${ }^{-}$is the highest while that of trans-SA and trans-SM ${ }^{2-}$ are similar.

In order to evaluate the possibility of the $\pi \pi^{*}-n \pi^{*}$ internal conversion, the $n \pi^{*}$ geometries of trans-SA, $\mathrm{SA}^{-}$and $\mathrm{SM}^{2-}$ are optimized and their vertical electronic transitions are calculated, as shown in Table S2. For trans-SA and $\mathrm{SM}^{2-}$, the $n \pi^{*}$ state is still described as the $\mathrm{S}_{2}$ state while the $\mathrm{S}_{1}$ state is the $\pi \pi^{*}$ state, indicating that the $n \pi^{*}$ state always lies in a higher energy level than the $\pi \pi^{*}$ state. Thus, the $\pi \pi^{*}-\mathrm{n} \pi^{*}$ internal conversion cannot occur. In contrast, the $\pi \pi^{*}-\mathrm{n} \pi^{*}$ internal conversion of trans- $\mathrm{SA}^{-}$is possible because the $n \pi^{*}$ state is more stable than the $\pi \pi^{*}$ state in the equilibrium $n \pi^{*}$ geometry of trans-SA${ }^{-}$. However, FTA spectra suggest that the excited-state evolutions of $\mathrm{SA}^{-}$and $\mathrm{SA}$ are very similar. In addition, in less polar organic solvents where the $n \pi^{*}$ state is more stable than in aqueous solution, ${ }^{23}$ there is no significant evidence to support the $\pi \pi^{*}-n \pi^{*}$ internal conversion. ${ }^{12}$ As a result, the role of the $n \pi^{*}$ state in the $\pi \pi^{*}$ state relaxations can be ignored. This is in a good agreement with the results that the hydration of $\mathrm{p}-\mathrm{MMC}$ impedes the $\pi \pi^{*}-\mathrm{n} \pi *$ internal conversion. ${ }^{24}$ 
Now that the $\pi \pi^{*}$-n $\pi^{*}$ internal conversion is unlikely to occur, in order to confirm the $\pi \pi^{*}$ relaxation through the trans-cis photoisomerization, the potential energy surfaces (PESs) of the photoisomerization of SA, $\mathrm{SA}^{-}$and $\mathrm{SM}^{2-}$ are constructed by LIIC method at the LR-PCM/TDM062X/6-31+g(d, p) calculation level, as shown in Figure 4. The isomerization paths of the three species are all barrierless, indicating that the photoisomerization occurs dynamically on PES, in agreement with the photoisomerization of the closely related p-MMC. ${ }^{6}$ Note that for every point in the three PESs, the $\mathrm{S}_{1}$ state is always the $\pi \pi^{*}$ state. The barrierless relaxation path is in good agreement with the ultrafast decays of $\pi \pi^{*}$ states, which are completed less than tens of picoseconds. Although TD-DFT is unable to locate the conical intersection (CI), it can give a qualitative description if the energy gap between the $\mathrm{S}_{0}$ and $\mathrm{S}_{1}$ states is small enough. ${ }^{25}$ Thus, the PESs shown in Figure 4 are believable. After initial photoexcitation, the $\pi \pi^{*}$ state evolves along the barrierless photoisomerization path characterized by the decreasing $\eta$. When the $\pi \pi^{*}$ state reaches the low-lying $\pi \pi^{*}-\mathrm{S} 0 \mathrm{CI}, \eta$ is $80^{\circ}$ for $\mathrm{SA}^{-}$and $90^{\circ}$ for both $\mathrm{SA}$ and $\mathrm{SM}^{2-}$. At this point, ultrafast nonradiative decay to the $\mathrm{S}_{0}$ state is extremely efficient. The system can either evolve to the cis-product or reverse back to the original trans-reactant. In addition, the reverse cis-trans isomerization is also barrierless, indicating that there is a dynamical equilibrium between the trans-cis and cis-trans reactions, similar to the case of $\mathrm{p}-\mathrm{MMC}{ }^{6}$ 


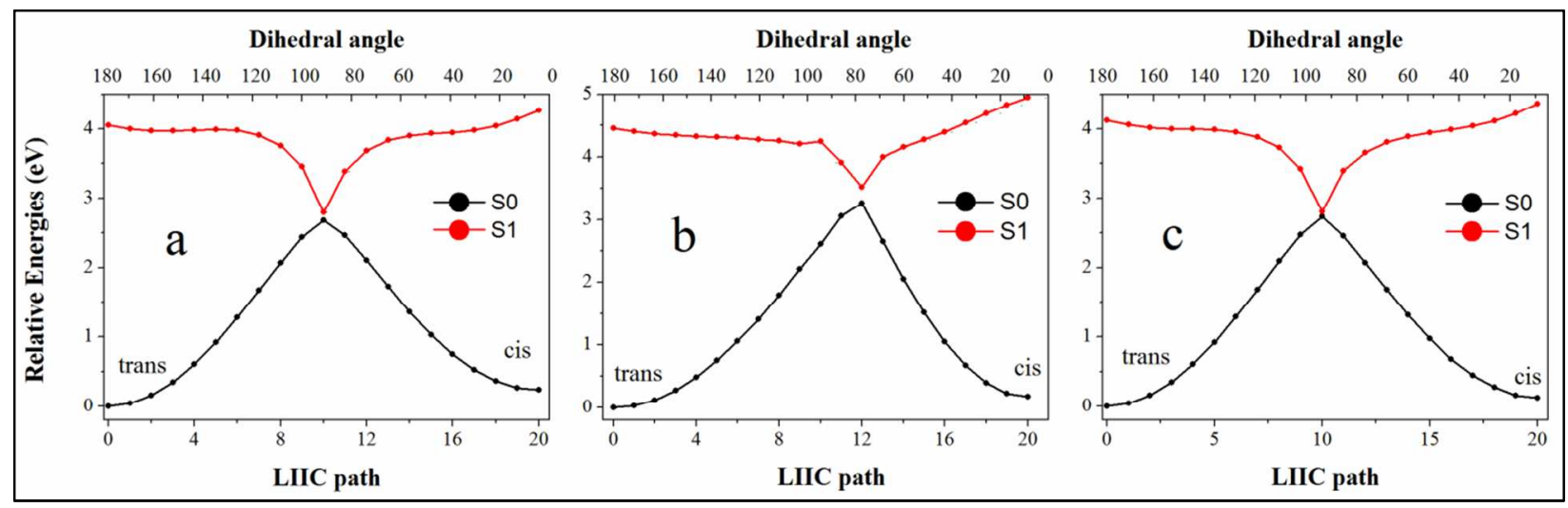

Figure 4. LR-PCM/TD-M062X/6-31+g(d, p) calculated linearly interpolated internal coordinate (LIIC) paths of the trans-cis photoisomerization of (a) SA, (b) $\mathrm{SA}^{-}$, and (c) $\mathrm{SM}^{2-}$. Dihedral angle $\eta\left(\mathrm{C}_{1}-\mathrm{C}_{2}-\mathrm{C}_{3}-\mathrm{C}_{4}\right)$ is also represented. In the original trans-reactant, the dihedral angle is $180^{\circ}$. And in the final cis-product, the dihedral angle is near to $0^{\circ}$.

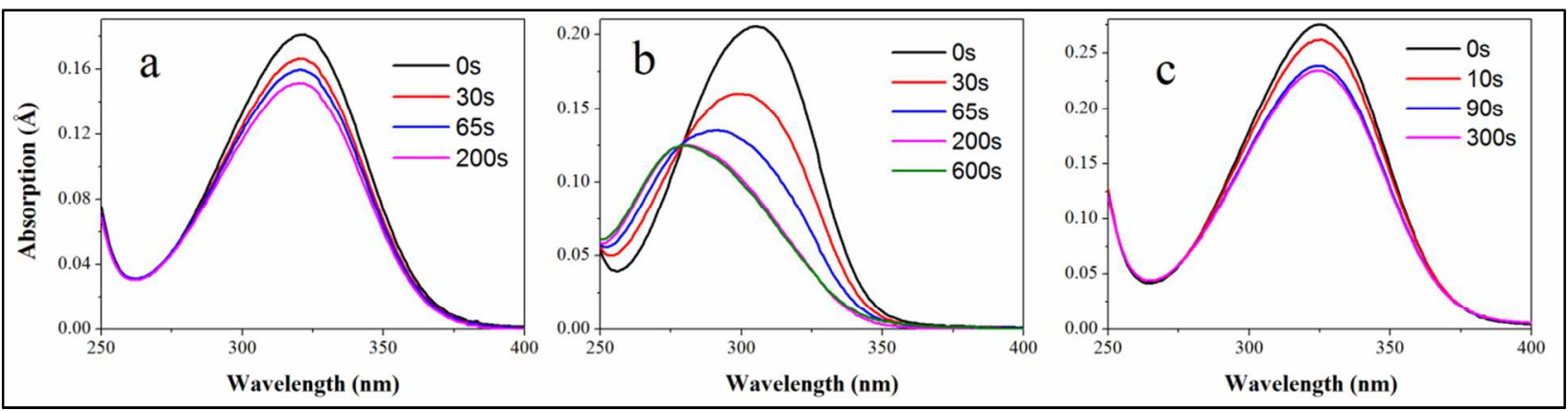

Figure 5. The absorption spectra of (a) $\mathrm{SA}$, (b) $\mathrm{SA}^{-}$, and (c) $\mathrm{SM}^{2-}$ after a period of $355 \mathrm{~nm}$ pulse irradiation.

As has been discussed, cis-photoproducts can be formed by nonradiative trans-cis photoisomerziation. ${ }^{12}$ To evaluate the effect of the formation of cis-products on the efficacy of sunscreen molecules, a continuous UV irradiation study is performed using the $355 \mathrm{~nm}$ pulse with a power of $3 \mathrm{~mW}$. Steady-state UV-Vis spectroscopy is used to probe the absorption changes induced by the formation of cis-photoproducts. As shown in Figure 5b, after $355 \mathrm{~nm}$ 
light irradiation, the absorption spectra of $\mathrm{SA}^{-}$are blue shifted significantly accompanied with largely decreasing intensities. This will largely reduce the efficacy of $\mathrm{SA}^{-}$as a sunscreen molecule. The spectral changes of $\mathrm{SA}^{-}$are in good agreement with the TD-DFT results shown in Table 2. TD-DFT results suggest that electronic excitation energy of cis-SA' is higher than that of trans-SA ${ }^{-}$and the oscillator strength of the former is smaller. As to SA and $\mathrm{SM}^{2-}$, the absorption spectra after the $355 \mathrm{~nm}$ light irradiation show no spectral shift and their intensities only decrease slightly, in agreement with the TD-DFT results that trans-SA and cis-SA have the same electronic excitation energies (Table 2). Although cis-SM ${ }^{2-}$ has $0.13 \mathrm{eV}$ higher excitation energy than trans-SM ${ }^{2-}$, the TD-DFT results is generally in good agreement with the irradiation results. As a result, $\mathrm{SA}$ and $\mathrm{SM}^{2-}$ are better sunscreen molecules than $\mathrm{SA}^{-}$.

In conclusion, we have studied the photoprotection mechanism of $\mathrm{SA}, \mathrm{SA}^{-}$and $\mathrm{SM}^{2-}$ in aqueous solution. The initially populated $\pi \pi^{*}$ states of the three species all decay to the $\mathrm{S}_{0}$ state nonradiatively via a barrierless trans-cis photoisomerization, which are completed in a few tens of picoseconds. The photoisomerization leads to the formation of cis-photoproducts. Cis-SA has much higher excitation energy and a decreased absorption cross section in the UV light than trans-SA${ }^{-}$. Thus, the efficacy of $\mathrm{SA}^{-}$as sunscreen is reduced largely after photoexcitation. In contrast, the absorption spectra of $\mathrm{SA}$ and $\mathrm{SM}^{2-}$ after photoisomerization just show slightly decreased intensities. These results suggest that $\mathrm{SM}^{2-}$ is a better sunscreen molecule than $\mathrm{SA}^{-}$, explaining nature's selection of sinapoyl malate rather than sinapic acid as the plant sunscreen. In addition, the protonated SA exhibits an ultrafast excited-state lifetime and its cis product still has a large absorption cross section on the UV region. An appropriate chemical modification on SA can make it a good sunscreen molecule used in neutral $\mathrm{pH}$ condition. These results highlight 
the role of the photoproduct in the efficacy of a sunscreen molecule, providing a new criterion for designing a new sunscreen molecule.

\section{ASSOCIATED CONTENT}

Supporting Information. Computational details, steady-state UV-Vis absorption spectra of sinapic acid and sinapoyl malate in $\mathrm{NaOH}$ solution, optimized ground-state geometries, vertical excitation energies of equilibrium $n \pi^{*}$ geometries. This material is available free of charge via the Internet at http://pubs.acs.org.

\section{AUTHOR INFORMATION}

\section{Corresponding Author}

*Email: klhan@dicp.ac.cn

\section{Notes \\ ${ }^{\#}$ These authors contributed equally to this work.}

The authors declare no competing financial interests.

\section{ACKNOWLEDGMENT}

We are grateful to the National Basic Research Program of China (2013CB834604) and the National Natural Science Foundation of China (Grant No: 21533010).

\section{REFERENCES}

(1) Gonzalez, S.; Fernandez-Lorente, M.; Gilaberte-Calzada, Y. The Latest on Skin Photoprotection. Clin. Dermatol. 2008, 26, 614-626.

(2) You, Y. H.; Lee, D. H.; Yoon, J. H.; Nakajima, S.; Yasui, A.; Pfeifer, G. P. Cyclobutane Pyrimidine Dimers Are Responsible for the Vast Majority of Mutations Induced by UVB Irradiation in Mammalian Cells. J. Biol. Chem. 2001, 276, 44688-44694. 
(3) Cadet, J.; Wagner, J. R. DNA Base Damage by Reactive Oxygen Species, Oxidizing Agents, and UV Radiation. Cold Spring Harb Perspect Biol. 2013, 5, 1-18.

(4) Baker, L. A.; Horbury, M. D.; Greenough, S. E.; Coulter, P. M.; Karsili, T. N. V.; Roberts, G. M.; Orr-Ewing, A. J.; Ashfold, M. N. R.; Stayros, V. G. Probing the Ultrafast Energy Dissipation Mechanism of the Sunscreen Oxybenzone after UVA Irradiation. J. Phys. Chem. Lett. 2015, 6, 1363-1368.

(5) Tan, E. M. M.; Hilbers, M.; Buma, W. J. Excited-State Dynamics of Isolated and Microsolvated Cinnamate-Based UV-B Sunscreens. J. Phys. Chem. Lett. 2014, 5, 2464-2468.

(6) Miyazaki, Y.; Inokuchi, Y.; Akai, N.; Ebata, T. Direct Spectroscopic Evidence of Photoisomerization in para-Methoxy Methylcinnamate Revealed by Low-Temperature MatrixIsolation FTIR Spectroscopy. J. Phys. Chem. Lett. 2015, 6, 1134-1139.

(7) Chang, X. P.; Li, C. X.; Xie, B. B.; Cui, G. L. Photoprotection Mechanism of p-Methoxy Methylcinnamate: A CASPT2 Study. J. Phys. Chem. A 2015, 119, 11488-11497.

(8) Kockler, J.; Oelgemoller, M.; Robertson, S.; Glass, B. D. Photostability of Sunscreens. J. Photochem. Photobio. C 2012, 13, 91-110.

(9) Chapple, C. C. S.; Vogt, T.; Ellis, B. E.; Somerville, C. R. An Arabidopsis Mutant Defectivein the General Phenylpropanoid Pathway. Plant Cell 1992, 4, 1413-1424.

(10) Ruegger, M.; Chapple, C. Mutations that Reduce Sinapoyl Malate Accumulation in Arabidopsis Thaliana Define Loci with Diverse Roles in Phenylpropanoid Metabolism. Genetics 2001, 159, 1741-1749.

(11) Fraser, C. M.; Chapple, C. The Phenylpropanoid Pathway in Arabidopsis. Arabidopsis Book 2011, 9, e0152.

(12) Baker, L. A.; Horbury, M. D.; Greenough, S. E.; Allais, F.; Walsh, P. S.; Habershon, S.; Stavros, V. G. Ultrafast Photoprotecting Sunscreens in Natural Plants. J. Phys. Chem. Lett. 2016, 7, 56-61.

(13) Dean, J. C.; Kusaka, R.; Walsh, P. S.; Allais, F.; Zwier, T. S. Plant Sunscreens in the UVB: Ultraviolet Spectroscopy of Jet-Cooled Sinapoyl Malate, Sinapic Acid, and Sinapate Ester Derivatives. J. Am. Chem. Soc. 2014, 136, 14780-14795.

(14) Vengris, M.; Larsen, D. S.; van der Horst, M. A.; Larsen, O. F. A.; Hellingwerf, K. J.; van Grondelle, R. Ultrafast Dynamics of Isolated Model Photoactive Yellow Protein Chromophores: "Chemical Perturbation Theory" in the Laboratory. J. Phys. Chem. B 2005, 109, 4197-4208.

(15) Smyk, B.; Drabent, R. Spectroscopic Investigation of Thee Quilibria of the Ionic Forms of Sinapic Acid. Analyst 1989, 114, 723-726.

(16) Allais, F.; Martinet, S.; Ducrot, P.-H. Straightforward Total Synthesis of 2-O-Feruloyl-Lmalate, 2-O-Sinapoyl-L-malate and 2-O-5-Hydroxyferuloyl-L-malate. Synthesis-Stuttgart 2009, 3571-3578.

(17) Yang B.; Yang, S. Temperature Effect on the Excited-state Dynamics 2-Methyl-5-t-butylp-quaterphenyl: Three-emission-state mode. Commun. Comput. Chem., 2015, 3, 112-122.

(18) Yang, S.; Han, K. Effects of Solvent Dielectric Constant and Viscosity on Two Rotational Relaxation Paths of Excited 9-(Dicyanovinyl) Julolidine. J. Phys. Chem. A 2016, 120, 49614965.

(19) Frisch, M. J. T.; G. W.; Schlegel, H. B.; Scuseria, G. E.; Robb, M. A. C., J. R., Scalmani, G.; Barone, V.; Mennucci,; B.; Petersson, G. A. N., H.; Caricato, M.; Li, X.; Hratchian, H.; et al. Gaussian 09, revision D.01; Gaussian Inc. Wallingford, CT,2009.

(20) Zhao, Y.; Truhlar, D. G. Density Functionals with Broad Applicability in Chemistry. Acc. Chem. Res. 2008, 41, 157-167. 
(21) Tomasi, J.; Mennucci, B.; Cammi, R. Quantum Mechanical Continuum Solvation Models. Chem. Rev. 2005, 105, 2999-3093.

(22) Cossi, M.; Barone, V. Time-Dependent Density Functional Theory for Molecules in Liquid Solutions. J. Chem. Phys. 2001, 115, 4708-4717.

(23) Liu, F.; Du, L.; Lan, Z.; Gao, J. Hydrogen Bond Dynamics Governs the Effective Photoprotection Mechanism of Plant Phenolic Sunscreens. Photochem. Photobiol. 2016. Doi: 10.1039/an9891400723.

(24) Miyazaki, Y.; Yamamoto, K.; Aoki, J.; Ikeda, T.; Inokuchi, Y.; Ehara, M.; Ebata, T. Experimental and Theoretical Study on the Excited-State Dynamics of ortho-, meta-, and paraMethoxy Methylcinnamate. J. Chem. Phys.2014, 141,244313.

(25) Gustavsson, T.; Banyasz, A.; Lazzarotto, E.; Markovitsi, D.; Scalmani, G.; Frisch, M. J.; Barone, V.; Improta, R. Singlet Excited-state Behavior of Uracil and Thymine in Aqueous Solution: A Combined Experimental and Computational Study of 11 uracil derivatives. J. Am. Chem. Soc. 2006, 128, 607-619. 


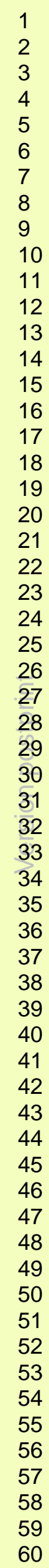

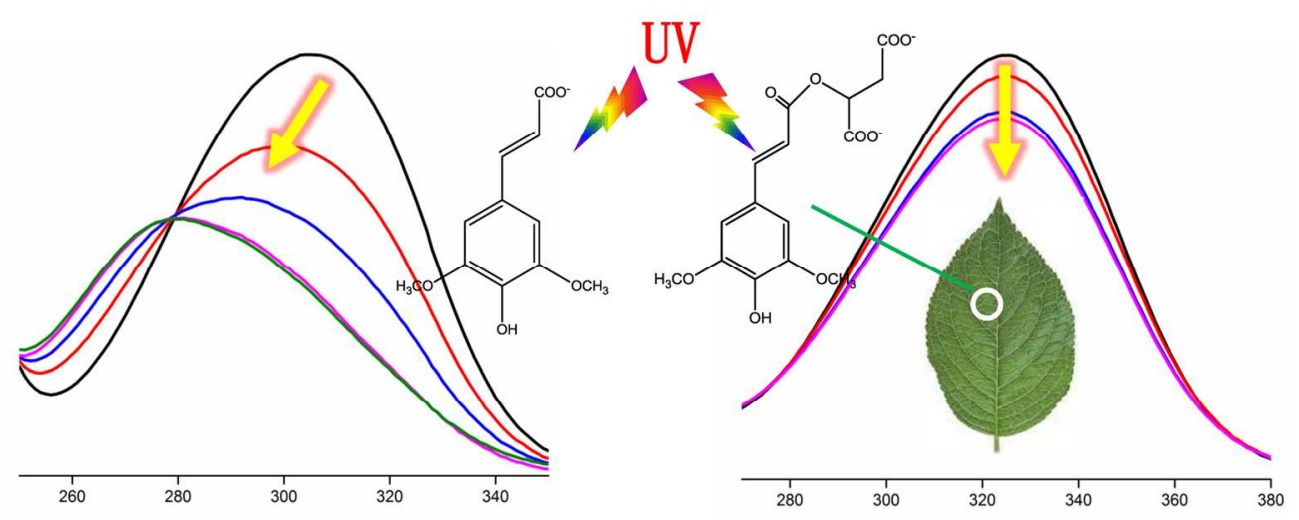

TOC

$76 \times 31 \mathrm{~mm}(600 \times 600 \mathrm{DPI})$ 